\title{
Statistics of magnetar crusts magnetoemission
}

\author{
V. N. KondratyeV ${ }^{1}$, Yu. V. Korovina ${ }^{2}$
}

${ }^{1}$ Physics Department, Taras Shevchenko National University of Kiev, 03022-UA Ukraine, and Bogolubov LTP, JINR, 141980, Dubna, Russia

${ }^{2}$ Moscow Institute of Open Education, 125167-RU Moscow, Russia

\begin{abstract}
Soft repeating gamma-ray (SGR) bursts are considered as magnetoemission of crusts of magnetars (ultranamagnetized neutron stars). It is shown that all the SGR burst observations can be described and systematized within randomly jumping interacting moments model including quantum fluctuations and internuclear magnetic interaction in an inhomogeneous crusty nuclear matter.
\end{abstract}

\section{Introduction}

The pioneering evidence for ultramagnetized astrophysical objects (magnetars) is associated with the March 5, 1979 event from SGR 0526-66 [1]. The magnetar concept, see $[2,3]$, is strongly corroborated by further observations of soft gamma repeaters (SGRs) indicating significant multipole magnetic fields being substantially larger than the dipole component $B_{\text {dip }} \sim 10^{15} \mathrm{G}$. For a noticeable contribution of magnetic pressure in a balance of crust forces in neutron star (NS) we write

$$
d B_{\text {crust }}^{2} / d R \approx 8 \pi \mathrm{GMn}(R) / R^{2},
$$

where the gravitational constant $\mathrm{G}$, and the star mass $M(R)$ within radius $R$ is related to the matter density $n(R)$ as $4 \pi R^{2} n(R)=d M / d R$. Substituting 
this relation into Eq. (1) and integrating over NS crust area we obtain the field strength $B \sim 10^{1.5}$ teratesla $\left(M / M_{o}\right)(10 \mathrm{~km} / R)^{2}$, with the solar mass $M_{o}$. Thus field torroidal components can reach tens teratesla (TT) that is consistent with estimates $[4,5]$ based on the supernova explosion energy. Such fields can affect the structure and properties of atomic nuclei [3-5]. In this contribution the randomly jumping interacting moments (RJIM) model [3] is further extended for an analysis of SGR bursts.

\section{Magnetodynamics of crusty nuclear matter}

The RJIM model for magnetodynamics simulations in NS crusts has already been described in [3]. We briefly remind that in simulations of demagnetization dynamics we use a very general form for magnetic moments $m$ of atomic nuclei $m=\mu \sum_{n} \nu_{n} \theta\left(b-b_{n}\right)=\mu I g$ with the nucleon magneton $\mu$, nuclear g-factor $g$ and spin $I$, as well as step function $\theta(x)$. Atomic nuclei occupied a volume $V_{\mathrm{D}}$ contribute to the magnetization $P=m / V_{\mathrm{D}}$. Taking $g=3$ for nuclear component of magnetic induction we get

$$
P=1.5 \text { TG } I n /\left(10^{13} \mathrm{~g} / \mathrm{cm}^{3}\right) \text {. }
$$

In a case of comparable sizes for nucleus and occupied volume $V_{\mathrm{D}}$ (i.e., $\left.n \sim 10^{13,5} \mathrm{~g} / \mathrm{cm}^{3}\right)$ internuclear interaction is ferromagnetic [3,6]. Taking for magnetic coupling strength between nearest-neighbor (nn) elements the value $J$ total Hamiltonian $\mathrm{H}$ for atomic nuclei array in a field $H$ can be expressed as follows $\mathrm{H}=-\sum_{i} b_{i} m_{i}$ through an interaction of magnetic moment $m_{i}$ with a local field $b_{i}=H(t)+J \sum_{j=\mathrm{nn}}+h_{i}$. Here the sum runs over nn elements and random fields $h_{i}$ with Gaussian distribution of a width $R$ called the disorder $[3,6-8]$, which allow to account for inhomogeneity, disorder and fluctuations.

\section{$3 \quad$ RJIM model for SGR-burst activity}

Let us consider adiabatically changing in time crust magnetic field $H$. When the local field value $b_{i}$ of a certain NS crust domain becomes less than certain value $b_{n}$ magnetization is changing stepwise. Due to the ferromagnetic interaction moment hopping may be triggered for nearest neighbors, which in turn may cause some discontinuity point for their neighbors, and so forth, producing, thereby, avalanches. Such avalanches lead to erratic jumps in magnetization curves and sharp release of magnetic energy estimated as

$$
E=H \Delta P V_{a}=10^{41} \operatorname{Ergs}(H / \mathrm{TT})\left(\Delta I n / 10^{13} \mathrm{~g} / \mathrm{cm}^{3}\right)\left(V_{a} / 10^{6} \mathrm{~m}^{3}\right) .
$$



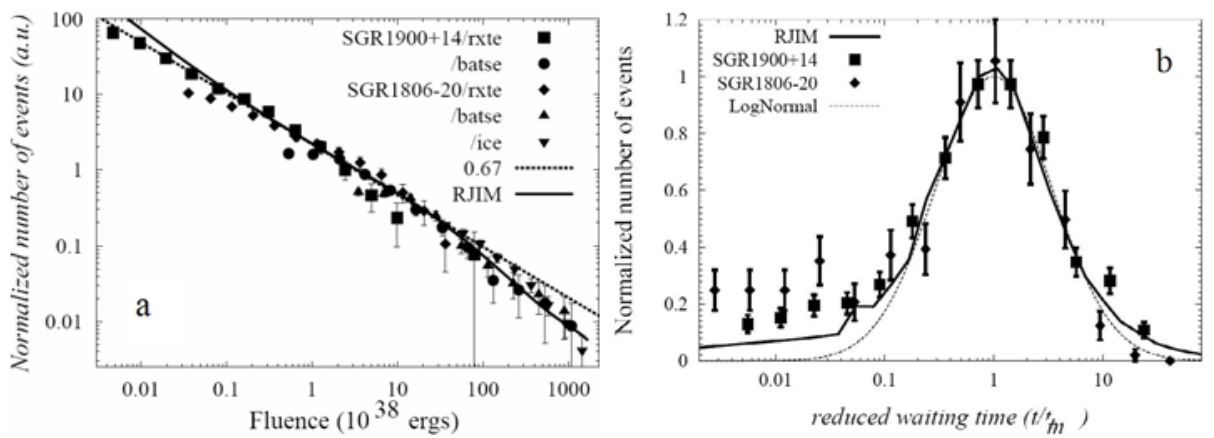

Figure 1: a) Normalized cumulative energy distributions of SGR-bursts are compared with the results of RJIM for the cubic lattice of a size $(150)^{3}$ represented by the solid line. The data of the RXTE and BATSE observations for SGR 1900+14 from [9] are shown by squares and circles, respectively. RXTE (diamonds), BATSE (up-triangles), and ICE (down-triangles) data for SGR 1806-20 are from [10]. The dashed line denotes the power law distribution. b) The reduced waiting time distribution between the successive RXTE/PCA bursts from SGR 1900+14 (squares) [9] and SGR1806-20 (diamonds) [10] are compared with the waiting time distribution between avalanches (solid curve). The dashed line represents the fit to the lognormal distribution of the width 3.6.

For a field strength $H \sim 3$ TT, typical magnitar crust density $n \sim 10^{13,5} \mathrm{~g} / \mathrm{cm}^{3}$, and avalanche linear size of order of outer NS crust thickness, $100 \mathrm{~m}$ (i.e., $V_{a}$ about $10^{6} \mathrm{~m}^{3}$ ) the amount of energy obtained from Eq. (3) is consistent with an energy of soft gamma-ray bursts.

Figure 1a represents the cumulative distributions of detected burst energy, i.e. the burst number with an energy exceeding certain value. The RJIM results are in a good agreement with observations for 7 periods of energy. The obtained event number dependence is well fitted by the power law with an exponent 0.67 , which corresponds to the value 1.67 for the differential distribution and provides a signal of self-organized criticality in the burst statistics.

Within RJIM model a time-scale in a period of burst activity is determined by the ratio of the disorder parameter $R$ and the field change rate $\tau=R /(d B / d t)$. Therefore, the scaling with respective time leads to an universal function as is shown in Fig. 1b for normalized waiting time, i.e., inter-burst time interval, distribution. 


\section{Conclusion}

We considered magnetodynamics of inhomogeneous crusty nuclear matter. Accounting for inter-nuclide magnetic coupling we show that quantum fluctuations in nuclear magnetic reactivity give rise to erratic jumps in magnetotransport of neutron star crusts. The resulting sharp energy releases lead to gamma-ray bursts. The properties of such a noise are favorably compared with burst statistics of Soft Gamma Repeaters. As is shown the predicted by RJIM model scaling properties for, e.g., the burst intensity and waiting time distributions, are in a good agreement with SGR observations supporting thereby the credibility of RJIM model. Thermal effects can also affect structural and collective nuclear matter properties, see [8].

This work is supported in part by scientific data center of Integral mission and SCOPES IZ73Z0-152485. One of us (V.N.K.) thanks JINR (Dubna, Russia) for warm hospitality and financial support.

\section{References}

[1] Mazets E.P. et.al. Nature (London) 282 (1979) 587.

[2] Svinkin D.S. et.al. Mon. Not. R. Astron. Soc. 447 (2015) 1028.

[3] Kondratyev V.N. Phys. Rev. Lett. 88 (2002) 221101.

[4] Kondratyev V.N. Phys. Atom. Nucl., 75 (2012) 1368.

[5] Kondratyev V.N. Eur. Phys. J. A 50 (2014) 7..

[6] Kondratyev V.N., Lutz H.O. Eur. Phys. J. D 9 (1999) 483.

[7] Kondratyev V.N. Phys. Lett. A 179 (1993) 209; Phys. Lett. A 190 (1994) 465.

[8] Kondratyev V.N., Di Toro M. Phys. Rev. C 53 (1996) 2176.

[9] Gogus E. et.al. Ap.J Letters 526 (1999) L93.

[10] Gogus E. et.al. Ap.J Letters 532 (2000) L121. 